\title{
Tree-Code modeling and addressing for non-ID physical objects in the Internet of Things
}

\author{
Huansheng Ning · Yang Fu • Sha Hu · Hong Liu
}

Published online: 9 October 2014

(C) The Author(s) 2014. This article is published with open access at Springerlink.com

\begin{abstract}
The Internet of Things (IoT) is an emerging network paradigm, and realizes the interconnection among ubiquitous things, along with its corresponding applications and services in both Physical-world and Cyber-world. Some researches have been worked on IoT modeling and addressing, which are mainly based on objects with a unique identifier (ID). However, in practical scenarios, there are several objects without any available ID, namely non-ID (i.e., nID) physical objects, including the objects unattached any ID itself, and the objects attached unreadable or un-trusted ID. It turns out that the nID physical objects related issues become noteworthy. In this paper, we focus on the nID physical objects to present a modeling and addressing solution. Concretely, (1) nID physical objects are described along with the discussion of applying nID solution; (2) a tree-like code structure (i.e., Tree-Code) is introduced to establish a unified modeling scheme for the nID physical objects according to abstracted data elements, and also achieves the compatibility with the ID physical objects considering the ID based data elements; (3) Tree-Code based addressing scheme is
\end{abstract}

\footnotetext{
H. Ning $(\varangle)$

School of Computer and Communication Engineering, University of Science and Technology Beijing, Beijing 100083, China

e-mail: ninghuansheng@ustb.edu.cn; ninghuansheng@buaa.edu.cn

Y. Fu $\cdot$ S. Hu

School of Electronic and Information Engineering,

Beihang University, Beijing 100191, China

e-mail: fary007@ee.buaa.edu.cn

$\mathrm{S}$. Hu

e-mail: husha89@gmail.com

H. Liu

Engineering Laboratory, Run Technologies Co., Ltd. Beijing,

Beijing 100192, China

e-mail: liuhong@bjrun.com
}

presented with dynamic updating considerations. It indicates that the nID physical object modeling and addressing provide complements to the ID physical objects for ubiquitous interactions in the IoT.

Keywords Internet of Things (IoT) - Object · Modeling · Addressing · Tree-Code

\section{Introduction}

The Internet of Things (IoT) is developing fast with increasingly worldwide attentions. The original IoT is thought to be a network paradigm in which all physical objects are tagged with radio frequency identification (RFID) transponder with a globally unique identifier (ID), i.e., electronic product code (EPC) [1]. Now IoT concept has been expanded and it's not limited to RFID technology. The vision for future IoT is ubiquitous, "everything connected, intelligent controlling, and anywhere covering". Towards IoT, studies have been worked on its models and frameworks [2-7]. The realization of future IoT will probably require dramatic changes in systems, architectures, communications and networking, especially identification for "everything".

As an important feature of future IoT, ubiquitous sensing and controlling lay the foundation for the mapping between Physical-world and Cyber-world, which is the prerequisite of IoT applications (e.g., intelligent living environment, and intelligent logistics) [8-10]. However, ubiquitous sensing alone is not enough for this mapping process, things' modeling and addressing are also important. Things' modeling is responsible for describing things with the data from ubiquitous sensors (e.g., RFID, radar, and video sensor), and it can be used for identification. Things' addressing has a wide range of meanings, such as determining the detailed informa- 
tion about the thing based on the modeling results, locating the things within a certain range, and even deducing or predicting future trend through combining the modeling results with the context information. There are two typical directions in things addressing process. For the first direction, things are queried from Physical-world and the addressing result exists in Cyber-world. While, for the second direction, things in Physical-world are queried from Cyber-world for some purpose (e.g., locating). For example, in the semantic web, computers can automatically query things (both physical objects and cyber entities) which are modeled by Ontology based languages [11], such as resource description framework (RDF). In some cases, both directions will combine with each other to accomplish a task.

With the development of computer science and Internet, Cyber-world has been proven to be helpful in modeling and addressing some limited physical objects (e.g., computers, routers, and servers) and cyber entities due to its nearly real time communication capability and strong computing power. Some examples (but not limited to) include IP address, domain name system (DNS), Web services, and search engine applications. The IoT was originally designated to model the physical objects with predefined code stored in a tag, and with the help of appropriate mechanisms such as ONS, the objects can be addressed for some purposes such as getting the objects' information and identifying the objects. In this paper, the above code based physical object modeling schemes are defined as ID based modeling schemes since ID is introduced for object modeling. Towards the ID based physical object modeling schemes, EPC [12] and Ubiquitous ID (UID) [13] are two representatives which utilize RFID technology for object identification. Current IoT applications (e.g., retail industry, and logistics) are mainly based on the ID-based systems [14-18].

After more than ten years development, the meaning of IoT has been greatly enriched, which refers to everything connected at anytime and in anyplace. Compared with the original RFID based objects modeling and addressing mechanisms, the adoption of multiple sensing technologies (e.g., RFID, and wireless sensor networks (WSN), smart mobile devices [19], and global positioning system (GPS)) have made the physical object modeling and addressing to be unprecedented complicated. Moreover, in many practical applications, there are objects without any available ID, called Non-ID (nID) physical objects. The nID physical objects include the objects unattached any ID itself, and objects attached unreadable or untrusted ID. In such cases, ID-based solutions become inapplicable. However, in IoT vision, identification of everything is significant for the realization of everything connected. Thus, it is urgent to explore new modeling and addressing schemes for physical objects without ID. Additionally, the uniform modeling and addressing for the ID and nID physical objects become the future trend in the IoT. In this paper, we are trying to solve the problem to model the physical objects by abstracting data elements, and to design the corresponding addressing mechanisms.

The main contributions of this paper are as follows: (1) introducing the concept of nID physical objects, and discussing the main significance of adopting nID modeling and addressing solutions; (2) proposing a unified modeling scheme (i.e., Tree-Code) for the nID physical objects in IoT applications which is also compatible with the ID based object modeling; (3) establishing a Tree-Code based addressing scheme, by which both ID and nID physical objects can be addressed in a unified form.

The rest of this paper is organized as following. Some related works are introduced in Sect. 2. Section 3 gives some preliminaries of nID physical objects. In Sect. 4, a Tree-Code structure is built for modeling the nID physical objects which also take ID data element into considerations. In Sect. 5, we give the addressing process for Tree-Code with dynamic updating mechanism specification. Section 6 concludes the paper and presents some open challenges.

\section{Related works}

\subsection{IoT modeling schemes}

In IoT, thing modeling refers to describe physical object and cyber entities in formal languages, and provides strong support for further addressing. Here, ontology has been introduced to establish reasonable description frameworks for things in the IoT. The variants of ontology provide a compatible understanding among different applications. Meanwhile, ontology can describe the heterogeneous data in semantic contexts, and address the data integration with unambiguous conceptualizations. The typical ontology languages including RDF, RDF schema (RDFS), and web ontology language (OWL), provide the normative syntax to describe entity classes/concepts, properties and relationships.

Thereinto, RDF as one of the most important modeling scheme, is originally developed by the World Wide Web Consortium (W3C), and this method can also used to describe the things in the IoT. RDF is based on eXtensible markup language (XML) that is a common syntax for the exchange and processing of metadata. XML syntax is a profile of standard generalized markup language (SGML), designed for Web to alleviate the implementation of the parser. XML syntax also guarantees the features such as vendor independence, extensibility, validation, and the ability to represent complex structures. RDF further extends the general XML syntax and model to provide unambiguous semantic expression, and to enable consistent encoding and exchange of standardized metadata. 
Concretely, RDF applies the common conventions to facilitate modular interoperability among heterogeneous metadata sets, in which standard mechanisms for representing semantics should be established based on a simple, yet powerful and rigorous, data model. Additionally, RDF provides both human-readable and machine-processable vocabularies to improve reuse and extension of metadata semantics among heterogeneous information. Vocabularies are the set of properties/attributes and metadata, and are defined by resource description communities to standardize the declaration of vocabularies.

Additionally, physical makeup language (PML) [20] raised by Auto-ID is intended to be a common "language" for describing physical objects, processes and environments. PML is another XML based markup language to describe physical objects and the corresponding relationships and interactions, and can also present resources ascribed by human. The main purpose of PML is to establish a general and standard method for formal modeling of objects, processes and environments in Physical-world. Based on PML, data structures and formats should be designed considering heterogeneous networks to provide efficient objects classification and generalization. Due to physical objects usually some degree of regularity and organization, PML mainly uses hierarchical organizational structure as data configuration. Therefore, PML includes a special data structure name to contain an exact name of an entity, and the name element includes multiple attributes to distinguish the different name representations. However, PML does not decide how to abstract the element from all the information available to model a physical object which is very important to the scalability and efficiency.

\subsection{IoT addressing schemes}

In IoT, addressing includes both physical object addressing and cyber entity addressing. Thereinto, the cyber entity addressing is similar to the addressing scheme in Internet based on domain names and IP addressing schemes. The physical objects addressing includes two aspects. One is the physical objects' mapping from Physical-world to Cyberworld, and the other refers to the same as the cyber entity addressing.

Towards physical object addressing, ONS is a typical approach to realize the interconnection between Physicalworld and Cyber-world. ONS presented by EPCglobal, is applied to access an object's information and its related services by EPC. Here, EPC is a universal identifier that provides a unique identity for a tagged physical object. ONS as an automated networking service, realizes the resource addressing in IoT, and also has similar functions to point each computer to Web sites. The main design idea is first to encode EPC into a fully qualified domain name (FQDN), and then to use DNS infrastructure to query for additional information. Concretely, in the case that a reader identifies a tag, the code is transmitted to an ONS on local networks or Internet via a middleware to find the stored information on the product. During the addressing, ONS points the middleware to a server where a file about that product is stored. The middleware retrieves the file, and the information about the product in the file can be forwarded to an inventory or other applications. Furthermore, EPC information services (EPCIS) is designed to enable EPC-related data sharing within and across enterprises, and the EPCIS standard defines standard interfaces to enable EPC-related data to be identified and subsequently to be queried with service operations and an associated data model. Such data interaction mainly based on persistent backend databases.

Towards cyber entity addressing, DNS is a typical addressing mechanism in Internet, which is a typical hierarchical naming system to connect computers, services, or resources into Internet or other private networks. Thereinto, a domain name is applied as an identifier to define a realm of autonomy, authority, or control on Internet. Domain names are formed by the rules and procedures of the DNS. Generally, a domain name represents an IP resource, and can be applied in various networking contexts and application-aware naming and addressing purposes.

\section{3 nID physical object preliminaries}

nID physical objects and nID-based IoT concepts were firstly introduced to model targets without any available ID in airport aviation risk management [6]. Here, nID physical objects definition is generalized and clarified for the ubiquitous objects in Physical-world that have no available ID but need to be identified, recorded, connected and operated. Note that many identification technologies have already existed based on varied information related to an object, such as space-time information, biometric characteristic, and other physical/chemical parameters.

- Space-time information can be jointly applied to determine ubiquitous objects. Thereinto, time information is always dynamically varied, and can be regarded as a unique parameter, and space information is also changed according to different positions. For instance, GPS [21] is a typical technology to detect the location information.

- Biometric characteristic based identification systems mainly applies the unique biometric attributes for identification, such as finger print recognition, hand geometry, face recognition, and retina/iris recognition [22]. The biometric characteristics can provide high identification accuracy with increasing applications. 
- Other physical/chemical parameters mainly refer to nonunique attributes including environmental temperature /humidity /vibration /pressure, object material /color /size, and radar cross section (RCS). For instance, the sensing technologies (e.g., radar, and infrared) are applied to detect such parameters for identification.

Many systems have been developed based on these technologies (e.g., fingerprint/iris based person recognition, electromagnetic scattering characteristic based object detection, and video based target identification). These technologies and systems have been proved to be the foundation of IoT. Towards above identification technologies, they are mainly dealing with the objects without any available ID tagged $[23,24]$. Moreover, these kinds of objects definitely take very important place in future IoT, which makes nID-based systems in IoT become an important topic, along with the corresponding modeling and addressing issues should be researched.

This paper focuses on unified modeling and addressing schemes for the ID/nID physical objects in Physical-world. The significance of applying nID solution includes:

- Enrich the physical object modeling methods in the IoT: Both ID physical objects and nID physical objects exist in IoT. ID physical objects are addressable via the modeling schemes of IP addresses, EPC/UID code, and other identifiers. By developing nID based modeling schemes, nID physical objects will also become addressable like ID physical objects, therefore combined modeling and addressing schemes can be established in the IoT.

- Realizing the things unity and fusion in the IoT: The heterogeneity of ubiquitous things impose great difficulty to their ID based modeling and addressing in IoT. The nID based modeling and addressing help to realize the unity and fusion of ubiquitous things through uniform machine understandable description.

- Improving transmitting efficiency: As most nID sensing data, such as data in video form, require large communication resources for transmitting, it is necessary to transmit the decreased data after modeling so that transmission efficiency can be improved.

- Reducing data storage: Since objects usually contain many elements and relations with other objects, storing their information directly will occupy large space. Assigning nID codes to them can reduce backend data storage.

nID physical object modeling and addressing are different with those of ID physical objects. It turns out that: (1) The ID physical object modeling is mainly based on an accurate identifier, which is more readable for identification and addressing; (2) The nID physical object modeling is mainly based on attributes, and the further addressing should be performed by using other algorithms (e.g., feature extraction, and fuzzy diagnosis).

\section{Tree-Code modeling for physical objects}

In IoT, physical objects can be "sensed" by many sensing techniques (e.g., vision monitor cameras, RFID, GPS, radar, and Infrared detector). When the object is sensed, the data element would be extracted from the objects' raw data. According to the type of objects and the sensing technologies, the data element can be classified into two categories: ID based data element and nID based data element. The ID based data element is an identifier allocated for objects artificially and previously that followed by a coding rule (e.g., bar code, and RFID code). For the nID based data element, the main purpose is to realize the identification of one or one class physical objects, and it should be under feature extraction and formalization before identifying an object. In this paper, we propose a physical object modeling scheme that support for the emerging nID data element and be compatible with the ID data element modeling.

\subsection{Data element abstraction for nID physical object modeling}

Towards the nID physical object modeling, nID data elements can be abstracted into three aspects: space-time information, attribute, and behavior.

- Space-time information: Considering the physical objects' boundary, each object in Physical-world has a unique location attribute at one moment in a coordinate system. In this paper, we use Location $(t)$ to represent the space-time information for a physical object. If the location is accurate and the detection interval is short enough to reach space-time consistency, objects' information at different time can be associated. In this case, their context information can also be associated. In some cases, an object can identified and addressed by only the Location $(t)$.

- Attribute: Besides the ID number and Space-time information, some kinds of sensors can detect object's nature attributes, such as color, shape, weight, and material. For instance, in the radar eyes, the object's attributes can be shown as RCS, glint, electromagnetic wave scattering sources, spatial power spectral, and Doppler. While a vision sensor can detect the object's vision attributes, color, size and shape. In most cases, the attributes can be used for object's category identification, not for unique. However, in some cases, the attributes can also be used to identify a thing uniquely. For instance, in the scenario of human recognition, when there is no ID based identifica- 


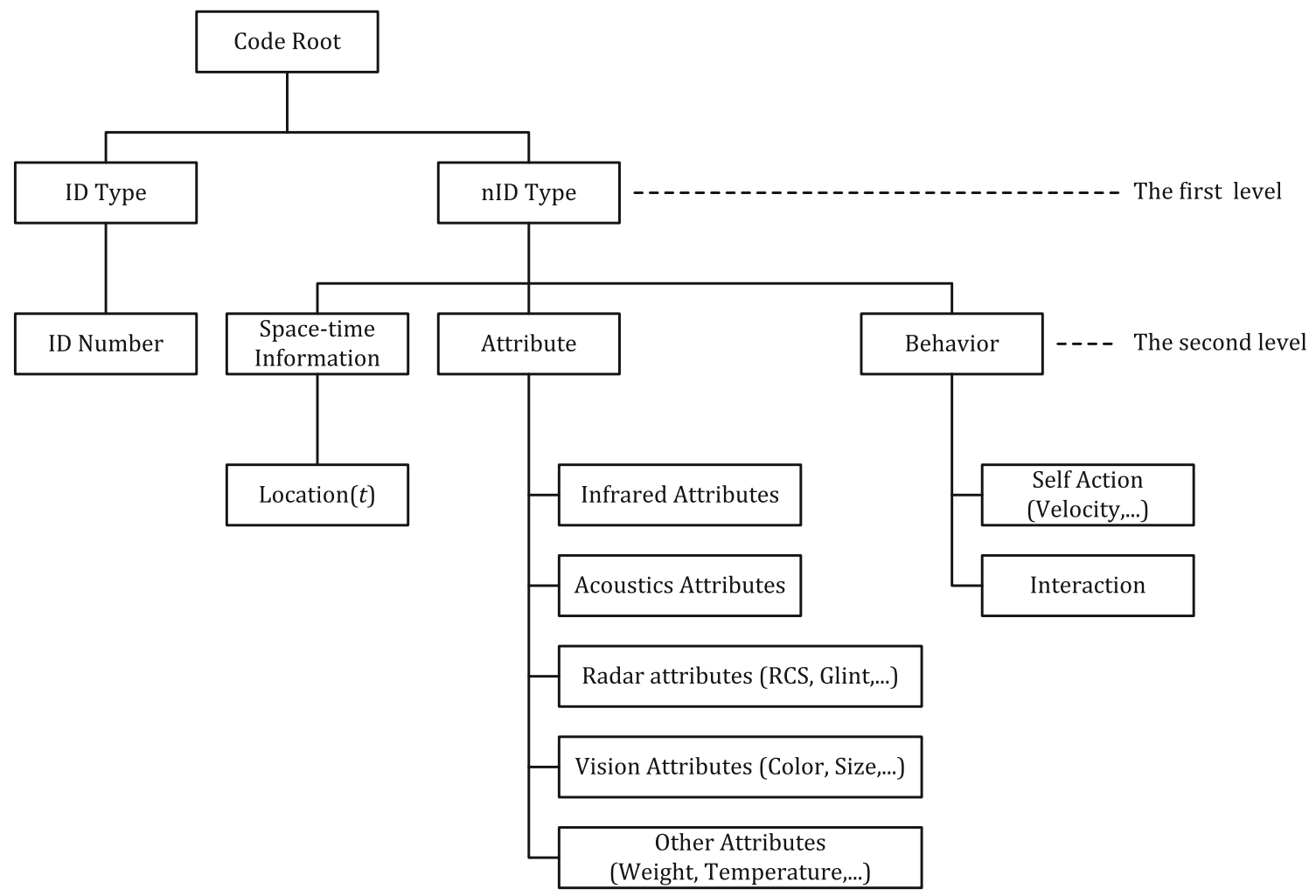

Fig. 1 The Tree-Code logical structure. Notice: this tree code structure is flexible which is convenient for adding high order levels, tree leaves, and extending new type in the 1 st level or 2nd level. The ID type is compatible with all existing coding rules in the world, e.g. bar code, and EPC code

tion, attributes of the person such as fingerprint, face, iris, and even DNA can be used and translated into codes to accomplish the identification uniquely.

- Behavior: The thing's behavior data means not only its self action, but also the interaction between two objects or the thing and environment it exists in. Meanwhile, behaviors can also be applied for identification, for instance a person can be identified according to the sensed walking postures.

\subsection{Tree-Code modeling with data elements}

The physical object modeling process is about how to use these data elements to describe the object efficiently. For physical object modeling, it is important to realize the universality of the modeling result, which means that the result can be understood by most of the users in the IoT. The modeling process is also responsible for dealing with the massive data element generated by sensing devices and reducing the data load transported on the network. In the following, a TreeCode structure is established to describe physical objects with ID/nID.

The Tree-Code structure (as shown in Fig. 1) is based on the abstracted data elements for the nID physical objects. The first level of Tree-Code is characterized with ID and nID type segment. The ID type segment aims to be compatible with all existing code types, e.g., quick response (QR) code, and EPC code. One or more code types can be supported by adding new ID type to the ID type segment to extend the modeling function.

The nID type segment in Tree-Code supports three branches: space-time information, attribute, and behavior. For the space-time information in Tree-Code, multiple position type should be included such as GPS, Galileo, Glonass, Beidou, or other satellite position system data format and even local positioning data types. Its value should better be dynamically updated with time. For attribute that is added into the Tree-Code, information about how to address it should also be added into the Tree-Code.

It is known that in RDF, web resource is described with the RDF triples (i.e., subject, predicate and object), and this resource modeling scheme can be borrowed into building Tree-Code with attribute. For example, when the attribute information gathered by a sensor is video picture, then the attribute can be modeled into the triple: subject (i.e., the target which is sensed), predicate (i.e., video picture), and object (i.e., the data of video picture). This triple can be used as a unit of the Tree-Code.

The behavior of physical objects may be in the form of self-behavior or interaction with other objects. The modeling of behavior is much more complicated than that of attributes which needs pretreatment to rationalize the relationship between the participants. After the pretreatment, 
the modeling of behavior can also be modeled into RDF triples. It is noteworthy that information about how to understand the behavior seems to be in great significance in building Tree-Code. Tree-Code is aimed to achieve universal and machine-readable modeling of physical objects. However, due to the diversity of sensing technologies and the corresponding addressing technologies, it is necessary for the Tree-Code to be able to provide extra information for the addressing systems' recognition. As a result, in Tree-Code, it not only offers the information about the content and name of the attribute/behavior, but also information about how to address it.

Sometimes, ID number alone cannot be used for everything's unique identification, for these cases, the combination of some nID elements can represent thing's unique identification, such as two objects with the same ID number but different space-time information. In other word, whether the Tree-Code can represent the thing's unique identification is determined by the data element it holds.

In some cases, the Tree-Code that is not unique is also useful or can satisfy the application's requirement. For example, in airport aviation avoiding birds-strike radar system, when radar is detecting and tracing a batch of birds, the detail serial number of birds is not critical. The bird size, bevy scale and flying direction, namely, nID elements, are important and sufficient to this application.

Based on the above Tree-Code structure, ID data element, space-time data element, attribute data element and behavior data element of a particular object are modeled into the TreeCode, and assigned to the corresponding ubiquitous objects. Different data elements can be incorporated in various applications.

\subsection{Tree-Code formal description}

The logical structure of Tree-Code above needs to be transformed into a specific data structure that can be understood by the machines in practical application for the automatic addressing process. Ontology, which has been studied for the modeling and description of resources in Semantic Web environment for many years, is helpful in modeling the objects and building the Tree-Code data structure. As a widely used Ontology based resource description method, RDF has been successfully used in the modeling and description of Web resources to realize the Semantic Web. RDF utilizes the Ntriple (i.e., resource, attribute, and attribute value) which can be taken as the subject-predicate-object expression to model and describe resource in Web. Moreover, Ontology and RDF can be introduced in Tree-Code. In the following, an example of RDF based Tree-Code object modeling is presented, as shown in Table 1. The object modeling in both scenarios is the same except for that in some cases the subjective in the latter scenario is unknown. Assume that in the multiple
Table 1 An example of tree-code data structure

\begin{tabular}{l}
\hline Algorithm \\
\hline$\langle$ Tree-Code root $\rangle$ \\
$\langle$ Tree-Code namespace $=$ “...” $\rangle$ \\
$\langle$ ID type $=E P C\rangle$ VALUE $\langle/ I D\rangle$ \\
$\langle$ Space-time information type $=$ GPS $\rangle$ Location $(t)$ \\
$\langle/$ Space-time information $\rangle$ \\
$\langle$ Attribure $\rangle$ \\
$\quad\langle$ Color $\rangle$ Red $;\langle/$ Color $\rangle$ \\
$\quad \ldots \ldots$ \\
$\langle/$ Attribute $\rangle$ \\
$\langle$ Behavior $\rangle$ \\
$\quad\langle$ Velocity $\rangle$ 8m/s; $\langle/$ Velocity $\rangle$ \\
$\langle$ Hold $\rangle$ Bag; $\langle$ Hold $\rangle$ \\
$\quad \ldots .$. \\
$\langle/$ Behavior $\rangle$ \\
$\langle/$ Tree-Code $\rangle$
\end{tabular}

sensors situation (as shown in Fig. 2). The object is assigned with an EPC identity, the RFID reader is embedded with GPS module, and the camera take a photo of the coming object. All the information is sent to the local modeling device for Tree-Code modeling.

\section{Tree-Code based addressing for nID physical objects}

In this Section, a typical Tree-Code based addressing scheme is established for nID Physical Objects, and the dynamic update mechanism is also discussed to enhance adaptivity.

\subsection{Tree-Code based addressing scheme}

Figure 2 shows a typical Tree-Code based addressing scheme, which includes two main parts: objects addressing and distributed application platform. The object addressing integrates two main functions modules:

- Addressing services: Addressing services can be classified into ID-based addressing services and nID-based addressing services according to the type of input, which is part of the Tree-Code. For ID-based addressing services, the addressing process is similar to that of ONS. However, the addressing process of nID-based servers is different. The interested resource may be distributed in multiple servers. A good choice for nID physical object addressing is taking advantage of the Content delivery network (CDN) [25], in which resource are addressed and routed by some piece of the content. CDN is suitable for addressing physical objects modeled by Tree-Code due to the abstracted data elements in Tree-Code. In some 
Fig. 2 The typical Tree-Code based addressing scheme

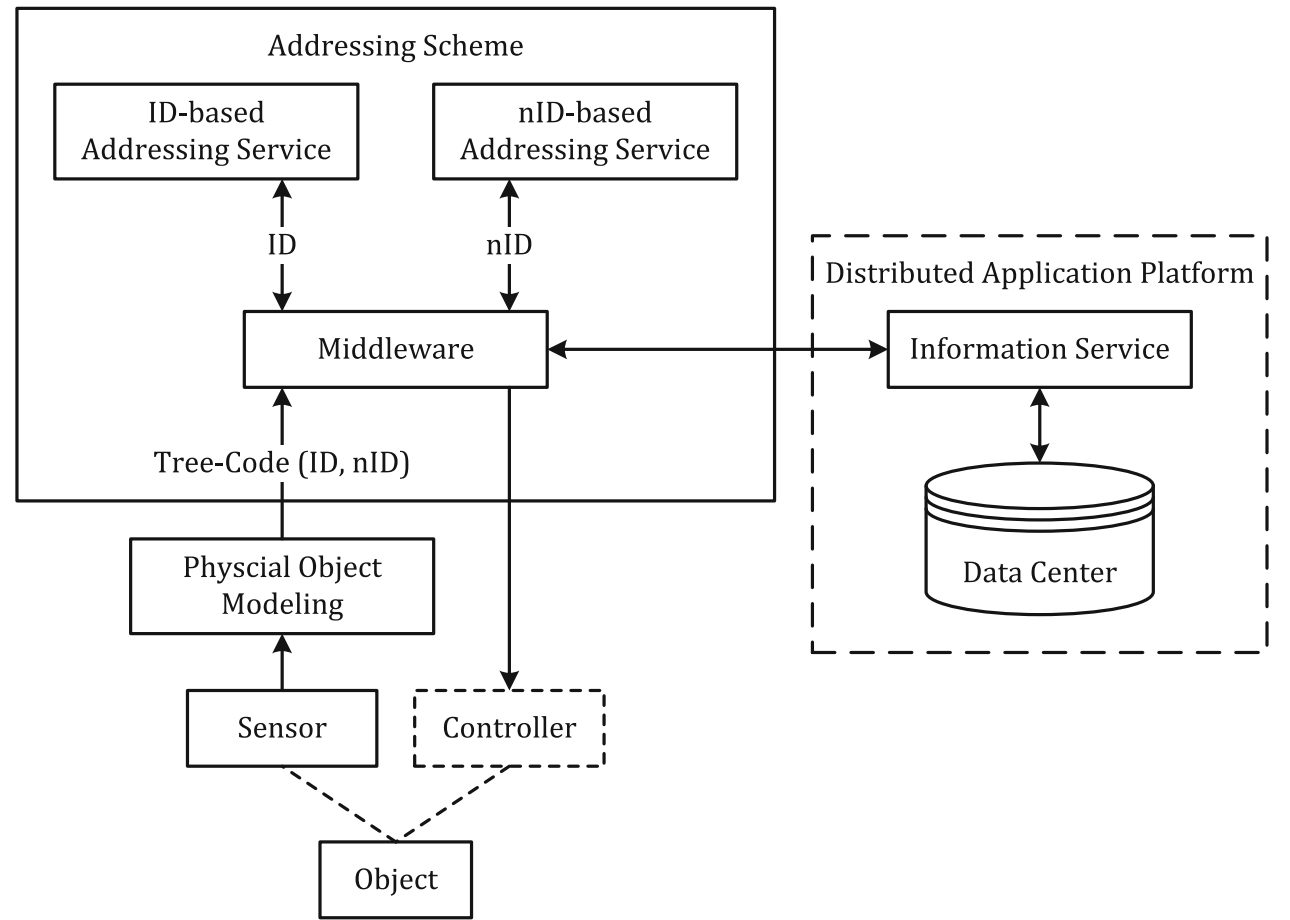

other applications, nID attribute (e.g., GPS based location data) had been used in designing routing protocols, which can be regarded as a special case of physical object addressing [26].

- Middleware: The middleware is a crucial function module for physical objects addressing which act as a conjunction that connects physical objects modeling, addressing services and distributed application platform. In the following, typical working modes of middleware are presented:

1. Physical to Cyber working mode: This working mode indicates the process that a physical object enters the sensing range of some ubiquitous sensors, instantly, its ID type data and nID type data are gathered and encoded into Tree-Code with the modeling rules we have discussed. The Tree-Code sent to middleware for further analysis after being constructed. The middleware can choose ID or nID based addressing services automatically. Then, the ID or nID codes are respectively transmitted to ID based or nID based addressing services for final addressing. The result of addressing services returns to middleware and is delivered to distributed application platform for further intelligent process or decision making. Finally, the feedback from distributed application platform will be transmitted to the middleware.

2. Cyber to Physical working mode: This working mode indicates that the physical objects addressing inquiry is launched from the Cyber-world (such as from distributed application platform), and the objective of the addressing process is to locate the physical objects. Similar to the Physical to Cyber working mode, the object that needs to been addressed is first modeled by Tree-Code, then the Tree-Code is send to the middleware which chooses addressing services automatically. The result from addressing services can be used by the middleware to locate a physical object based on which the data (e.g., controlling command) will be sent to it. ;

3. Hybrid working mode: In some cases, the middleware works as the combination of the above working modes which is named as hybrid working mode here due to the fact that this working mode composes both the direction from Physical-world to Cyber-world and from Cyber-world back to Physical-world. Loop working mode is common in context aware and ubiquitous computing applications in IoT, in which physical objects together with the context information is modeled with Tree-Code. The middleware, addressing services, and the distributed application platform works together to return controlling command to the Physical-world.

In the above typical addressing scheme, distributed application platform focuses on specific application oriented intelligent functions, such as decision-making, intelligent controlling, and data managing. The distributed application platform 
directly interacts with the middleware. The interaction can be triggered by either the distributed application platform or the middleware. In order to make better illustration of the distributed application platform, an example on bank monitoring scenario is given as follows. Assume that there is an RFID reader and a camera installed at the entrance of the ATM (Automated Teller Machines) room, if a person comes, the video picture and the ID are acquired respectively by the camera and the RFID reader. The data is modeled into TreeCode and sent to the middleware, the middleware automatically chooses addressing services for the Tree-Code. After addressing process, the related result will be sent to the bank security application platform for further analysis. If the person is with criminal record, the application platform will then send an alarm to the security department of the bank to make sure that they pay attention to that person.

\subsection{Dynamic update mechanism in nID physical object addressing}

The modeling of nID based physical objects is based on the sensing data of ubiquitous sensors. In some cases where the physical object is with strong dynamicity, dynamic updating mechanism is needed to ensure satisfying addressing result. However, the frequent updating of object's modeling result will cause severe problems such as network traffic load increase, energy wastes and so on. As a result, attention should be paid to the dynamic updating mechanism.

In the following, we propose a self-tuning and userinvolving updating scheme. In this scheme, the addressing servers maintain a prediction model for the modeling results with dynamic sensing data. The prediction model is created when the modeling result is first generated. In order to achieve the predicting goal, two issues have to be addressed: the first is how to find the dynamic data element in the Tree-Code, and the second is how to build prediction models for the data element. The first issue is addressed through comparing the Tree-Code of an object that generated at different times. The second issue can be addressed by some algorithms such as stochastic based model, time series based model, and algorithmic based model. The prediction model is self-tuning, and the accuracy index of the model will evolve with the addressing process. The user (whoever sends the addressing request to the addressing server, no matter a human or a machine) set the standard for the addressing process, and the addressing server make decision on whether to update the object modeling result in it based on the user's standard and the accuracy index of the prediction model. For example, if the user's standard is under the accuracy index, an operation update is unnecessary. Otherwise it must execute the updating operation.

\section{Conclusions and challenges}

In this paper, we have clarified the nID physical objects definition and the main significance. Based on nID physical objects' basic data elements (i.e., space-time information, attribute, and behavior), the nID physical objects together with the ID physical objects are modeled into Tree-Code. Then a typical addressing scheme which mainly includes addressing services, middleware and distributed platform is demonstrated. Due to the dynamicity of Tree-Code which is caused by the objects' changing status, dynamic update mechanism in nID physical object addressing is discussed. The nID-based IoT system implementation needs other technologies besides modeling and addressing introduced in this paper, and challenges shall be further considered, including:

1. Heterogeneous sensing data fusion: as multiple and heterogeneous sensors are involved in IoT, Cooperative modeling scheme is necessary to integrate nID and ID date element, which is significant for the integration of ID/nID-based IoT systems.

2. Addressing time control: nID physical objects addressing involves multiple servers, the addressing time for servers vary in a wide range due to different task complexity, processing ability and communicating bandwidth. The addressing time control has to find the optimal trade-off between performance and waiting time.

3. Space-time consistency: in some cases, the object status is changing all the time, beside, due to the deviation in both time and space dimension, the objects' modeling and addressing will suffer performance degradation. So space-time consistency is also a challenge to physical objects' modeling and addressing.

Acknowledgments This work was funded by the National Natural Science Foundation of China (61471035), and also supported by the DNSLAB, China Internet Network Information Center, Beijing 100190, China.

Open Access This article is distributed under the terms of the Creative Commons Attribution License which permits any use, distribution, and reproduction in any medium, provided the original author(s) and the source are credited.

\section{References}

1. Welbourne, E., Cole, G., Gould, K., Rector, K., Raymer, S., Balazinska, M., et al. (2001). Building the internet of things using RFID: The RFID ecosystem experience. IEEE Internet Computing, 13(3), 48-55.

2. Ashraf, U., Abdellatif, S., \& Juanole G. (2011). Route selection in IEEE 802.11 wireless mesh networks, Telecommunication Systems. Retrieved October 10, 2012 from http://link.springer.com/ content/pdf/10.1007\%2Fs11235-011-9493-5. 
3. Atzori, L., Iera, A., \& Morabito, G. (2011). SIoT: Giving a social structure to the internet of things. IEEE Communications Letters, 15(11), 1193-1195.

4. Dillon, T. S., Zhuge, H., Wu, C., Singh, J., \& Chang, E. (2011). Web-of-Things framework for cyber-physical systems. Concurrency And Computation-Practice \& Experience, 23(9), 905-923.

5. Ma, J., Wen, J., Huang, R., \& Huang, B. (2011). Cyber-Individual meets brain informatics. IEEE Intelligent Systems, 26(5), 30-37.

6. Ning, H., Hu, S., He, W., Xu, Q., Liu, H., \& Chen, W. (2012). Non-ID based internet of things and its application in aviation risk management. Chinese Journal of Electronics, 21(2), 209-214.

7. Zhong, N., Ma, J., Huang, R., Liu, J., Yao, Y., Zhang, Y., \& Chen, J. (2010). Research challenges and perspectives on Wisdom Web of Things (W2T). Journal of Supercomputing, Retrieved October 10, 2012 from http://dx.doi.org/10.1007/s11227-010-0518-8.

8. Chiu, K. L., \& Hwang, R. H. (2012). Communication framework for vehicle ad hoc network on freeways. Telecommunication Systems, 50(4), 243-256.

9. Meer, H., Hummel, K. A., \& Basmadjian, R. (2012). Future Internet services and architectures: trends and visions. Telecommunication Systems, 51(4), 219-220.

10. Zhang, D., Yang, L. T., \& Huang, H. (2011). Searching in internet of things: Vision and challenges. In Proceedings of IEEE Ninth International Symposium on Parallel and Distributed Processing with Applications (pp. 201-206). IEEE.

11. Daouadji, A., Nguyen, K. K., Lemay, M., \& Cheriet, M. (2010). Ontology-based resource description and discovery framework for low carbon grid networks. In Proceedings of 2010 First IEEE International Conference on Smart Grid Communications (pp. 477482). IEEE.

12. David, L. (2005). The electronic product code (EPC). Cambridge: MIT Auto-ID Center Massachusetts Institute of Technology.

13. Sakamura, K., \& Koshizuka, N. (2005). Ubiquitous computing technologies for ubiquitous learning. In Proceedings of the 2005 IEEE International Workshop on Wireless and Mobile Technologies in Education (pp. 11-20).

14. Hong, S., Kim, D., Ha, M., Bae, S., Park, S. J., Jung, W., et al. (2010). SNAIL: An IP-based wireless sensor network approach to the Internet of Things. IEEE Wireless Communications, 17(6), $34-42$.

15. Kong, N., Li, X., \& Yan. B. (2008). A model supporting any product code standard for the resource addressing in the Internet of things. In Proceedings of the 1st International Conference on Intelligent Networks and Intelligent Systems (pp. 233-238).

16. Lampropoulos, K., \& Denazis, S. (2011). Identity management directions in future internet. IEEE Communications Magazine, 49(12), 74-83.

17. Roussos, G., \& Chartier, P. (2011). Scalable ID/locator resolution for the IoT. In Proceedings of 2011 IEEE International Conference on Internet of Things, and 4th IEEE International Conference on Cyber, Physical and Social Computing (pp. 58-66).

18. Xu, B., Liu, Y., He, X., \& Tao, Y. (2010). On the architecture and address mapping mechanism of IoT. In Proceedings of the 2010 IEEE International Conference on Intelligent Systems and Knowledge Engineering (pp. 678-682). IEEE.

19. Koo, B., Han, K., James, J., \& Park, T. S. (2011). Design and implementation of a wireless sensor network architecture using smart mobile devices. Telecommunication Systems. Retrieved October 10, 2012 from http://link.springer.com/content/pdf/10. 1007\%2Fs11235-011-9535-z.
20. Brock, D. L. (2011). The physical markup language-A universal language for physical objects. Retrieved October 10, 2012 from http://wew.autoidlabs.org/uploads/media/MIT-AUT OID-WH-003.

21. Hofmann-Wellenhof, B., Lichtenegger, H., \& Collins, J. (1997). Global positioning system: Theory and practice (4th ed.). Vienna: Springer.

22. Jeong, G. M., Ahn, H. S., Choi, S., Kwak, N., \& Moon, C. (2010). Pattern Recognition Using Feature Feedback: Application to Face Recognition. International Journal of Control, Automation, and Systems, 8(1), 141-148.

23. Munoz-Organero, M., Ramíez-González, G. A., Munoz-Merino, P. J., \& Delgado Kloos, C. (2010). A collaborative recommender system based on space-time similarities. IEEE Pervasive Computing, 9(3), 81-87.

24. Till, Q., Herbert, B., \& Gool, L. (2008). Object recognition for the internet of things. In Proceedings of the 1st International Conference on the Internet of Things (pp. 230-246).

25. Morikawa, H., \& Aoyama, T. (2004). Realizing the ubiquitous network: the internet and beyond. Telecommunication Systems, 25(3,4), 449-468.

26. Wang, S. T., \& Wu, J. L. C. (2004). SABAGAR: A simple attributebased addressing and GPS-aided routing protocol for applications in wireless sensor networks. Telecommunication Systems, 26(2-4), $197-212$

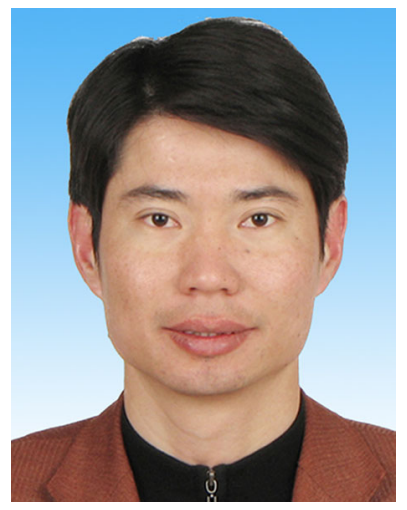

Huansheng Ning received a B.S. degree from Anhui University in 1996 and Ph.D. degree in Beihang University in 2001. Now, he is an Professor in School of Computer and Commnication Engineering, University of Science and Technology Beijing, China. His current research focuses on Internet of Things, Cyber-Physical Modeling, Electromagnetic Sensing. He serves as an associate editor of IEEE System Journal and IEEE Internet of Things Journal.

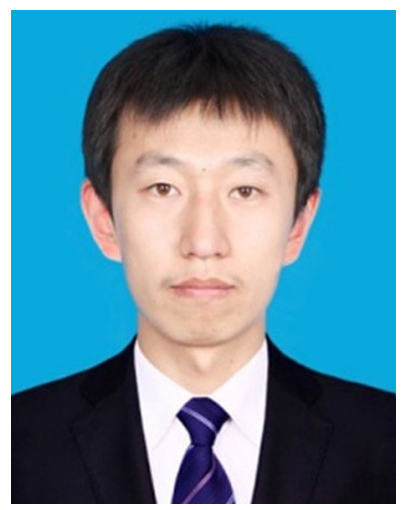

Yang $\mathbf{F u}$ received the B.E. degree from School of Information Science and Technology, Northeastern University, China. Now he is a M.S. candidate in School of Electronic and Information Engineering, Beihang University, China. His research interests are energy management and Internet of Things. 


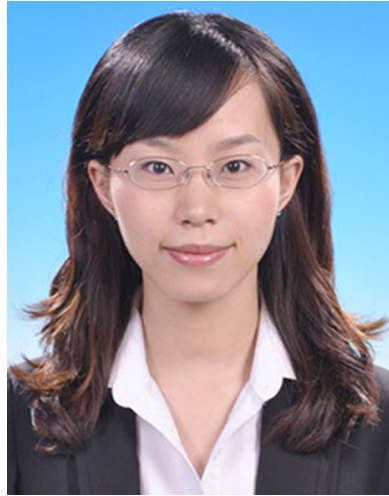

Sha Hu received the B.E. degree from International School, Beijing University of Posts and Telecommunications. Now she is a M.S. candidate in School of Electronic and Information Engineering, Beihang University, China. Her research interests are time-space consistency and Internet of Things.

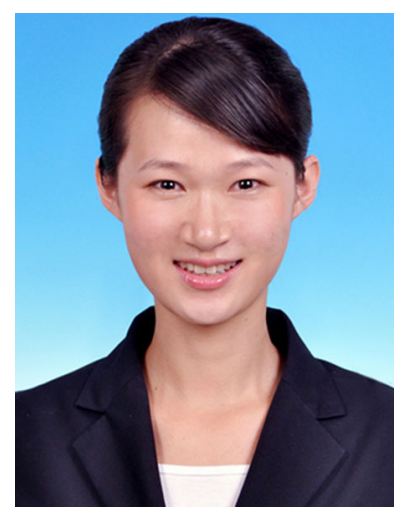

Hong Liu received a Ph.D. degree at the School of Electronic and Information Engineering, Beihang University, China. Currently, She is a research fellow in Engineering Laboratory, Run Technologies Co., Ltd. Beijing, China. She focuses on the security and privacy issues in radio frequency identification, vehicle-to-grid, and Internet of Things. Her research interests include authentication protocol design, and security formal modeling and analysis. 